\title{
CARTILHA DE ORIENTAÇÕES NUTRICIONAIS PARA PORTADORES DE DIABETES E HIPERTENSÃO ARTERIAL SISTÊMICA
}

\section{PRIMER NUTRITIONAL GUIDELINES FOR CARRIERS OF DIABETES AND HYPERTENSION}

\author{
Márcia Ribeiro Braz ${ }^{1}$, Paula Balbi M. Hollanda Cordeiro ${ }^{2}$, Fábio Aguiar Alves ${ }^{3}$, Adilson \\ da Costa Filho ${ }^{4}$ \\ ${ }^{1,2,4}$ Centro Universitário de Volta Redonda/ Mestrado Profissional em Ensino de Ciências da Saúde e do \\ Meio Ambiente/marciabraz2009@gmail.com/erikivf@ig.com.br/ \\ adilson.costa.filho@gmail.com \\ ${ }^{3}$ Universidade Federal Fluminense/Departamento de Epidemiologia faalves@gmail.com
}

\section{Resumo}

O agente comunitário de saúde é o condutor do conhecimento no Programa Estratégia Saúde da Família, pois é ele quem faz visitas domiciliares, conhecendo, dessa forma, o estilo de vida daquela comunidade, sua história e comprometimentos de saúde. Em face da progressiva expansão do processo de organização dos serviços de atenção básica nos municípios, este profissional necessita de programas e conteúdos que o possibilite a desempenhar suas atribuições, cada vez mais próximo das necessidades de saúde da população. Portanto, este estudo tem como principal objetivo construir uma cartilha de ensino e capacitação, de fácil interpretação, para instruir o agente comunitário na atenção nutricional direcionada a portadores de Diabetes Mellitus e Hipertensão Arterial Sistêmica. Pretende-se, também, através da implantação desse instrumento educacional disseminar o conhecimento básico sobre diabetes e hipertensão, visando à modificação do padrão alimentar da população assistida. Entendemos que a cartilha "Orientações Nutricionais para a Estratégia Saúde da Família - Diabetes e Hipertensão", desenvolvida neste estudo venha ajudar às equipes de saúde da Atenção Primária, principalmente o agente comunitário de saúde, a orientar a comunidade no que diz respeito a alimentação de diabéticos e hipertensos.

Palavras-chave: Ensino em saúde, Cartilha educacional, Agente comunitário

\begin{abstract}
The community health care agent is the driver of the knowledge in the Family Health Program, this is who makes home visits, knowing thus the lifestyle of that community, its history and health impairments. Given the gradual expansion of the process of organizing primary care services in the municipalities, this professional needs programs and contents that allows one to perform their duties, increasingly close to the health needs of the population. Therefore, this study has as main objective to build a primer education and training, easy to interpret, to instruct the agent community in the targeted nutritional care to patients with Diabetes Mellitus and Hypertension. It is intended, also, through the implementation of this educational tool to disseminate basic knowledge about diabetes and hypertension, aimed at changing the nutrition patterns of the assisted population. We understand that the book "Nutritional Guidelines for the Family Health Diabetes and Hypertension," developed in this study will help teams of primary health care, particularly the community health agent, guiding the community in relation to feeding diabetes and hypertension.
\end{abstract}

Key words: Learning in health, Education folder, community health agent. 


\section{Considerações Iniciais}

No contexto epidemiológico nacional atual, de acordo com o Instituto Brasileiro de Geografia e Estatística - IBGE (2006) e o Ministério da Saúde (BRASIL, 2008), relevantes mudanças socioeconômicas, geográficas, políticas e tecnológicas ocorreram nos últimos 50 anos, com consequentes transformações nas relações de trabalho, nas formas de oferta e procura pelos serviços públicos na atividade física e no gasto energético, no lazer e em outros aspectos. Mudanças ainda mais drásticas ocorreram nas formas de se produzir, comercializar, transformar, industrializar e preparar os alimentos. Ainda, de acordo com essas fontes, como consequência desse processo, cresce o consumo de alimentos calóricos, com alto teor de açúcares, gorduras, sal e aditivos químicos, que são pobres em nutrientes como vitaminas, sais minerais e fibras. Essa ação está associada ao baixo consumo de frutas, legumes e verduras, e é hoje o padrão mais comum de alimentação das famílias brasileiras.

\footnotetext{
Essas transformações resultaram em alterações significativas no perfil de morbidade e mortalidade e nos padrões do consumo alimentar e do estilo de vida da população, determinando os fenômenos das denominadas transições demográfica, epidemiológica e nutricional, bem documentados no país em diversas publicações (BATISTA et al, 2007).
}

O Conselho Federal de Nutricionistas - CFN - afirma que a transição nutricional no Brasil é marcada pela dupla carga de doenças, com a convivência de doenças infecciosas e transmissíveis, desnutrição e carências nutricionais específicas e de doenças crônicas não transmissíveis (DCNT) relacionadas à alimentação, tais como Obesidade, Hipertensão Arterial (HÁ), Doenças Cardiovasculares, Diabetes Mellitus (DM) e alguns tipos de Câncer, em todas as faixas de renda da população, em particular, entre as famílias de menor poder socioeconômico (CFN, 2010).

Passos (2006) afirma que a hipertensão é a mais frequente das doenças crônicas não transmissíveis e o principal fator de risco para complicações cardiovasculares como acidente vascular cerebral e infarto agudo do miocárdio, além da doença renal crônica terminal. Segundo a Sociedade Brasileira de Cardiologia (SBC), a alta prevalência e as consequências sociais e econômicas da hipertensão arterial sistêmica a caracterizam como um problema de saúde pública no Brasil (LONGO, et al., 2009). Os mesmos autores prosseguem dizendo que, em 2006, 17 milhões de brasileiros apresentavam hipertensão, o que representava 35\% dos indivíduos adultos com mais de quarenta anos. 
A prevalência do diabetes, assim como a da HA também vem crescendo mundialmente e configura-se atualmente como uma epidemia resultante, em grande parte, do envelhecimento da população. O sedentarismo, a alimentação inadequada e o aumento da obesidade também são responsáveis pela expansão global do diabetes. As hospitalizações atribuíveis ao Diabetes Mellitus representam 9\% dos gastos hospitalares do Sistema Único da Saúde (ROSA \& SCHMIDT, 2008). Wild et al. (2004), em seu estudo, mencionam que a Organização Mundial da Saúde (OMS) estimou que até o ano de 2030 o Brasil terá 11,3 milhões de diabéticos.

Dentre o conjunto de prioridades políticas apresentadas pelo Ministério da Saúde (BRASIL, 2008) e aprovadas pelo Conselho Nacional de Saúde (CNS), destacam-se a expansão e a qualificação da atenção básica, organizadas pela Estratégia Saúde da Família (ESF). Uma concepção que se desenvolve por meio de práticas gerenciais e sanitárias, democráticas e participativas, sob a forma de trabalho em equipes, dirigidas às populações de territórios delimitados, pelos quais assumem responsabilidades. Essa prática supera a antiga proposição de caráter centrado exclusivamente na doença.

As equipes da ESF são compostas, no mínimo, por um médico de família, um enfermeiro, um auxiliar de enfermagem e 6 agentes comunitários de saúde (ACS). Quando ampliada, conta ainda com um dentista, um auxiliar de consultório dentário e um técnico em higiene dental. O trabalho das equipes é o elemento-chave para a busca permanente de comunicação e troca de experiências e conhecimentos entre os integrantes da equipe e desses com o saber popular do ACS (BRASIL, 2008).

Acreditamos que o ACS é o elemento chave da equipe da ESF junto à comunidade, pode desenvolver ações preventivas no campo das doenças crônicas não transmissíveis, como o Diabetes e a Hipertensão Arterial.

Na região Sul Fluminense do estado do Rio de Janeiro, o ACS é o sujeito de viabilização de políticas de saúde de uma nova linhagem e seu trabalho, de modo geral, ultrapassa $\mathrm{o}$ atendimento às necessidades, pois se dedica a cuidar da comunidade $\mathrm{e}$ pensa a saúde em sua concepção ampliada. Cuidar é mais que tratar o indivíduo nas unidades de saúde, é prover assistência na vida comunitária, deslocando o cuidado para o território onde se insere a população adstrita.

O entendimento de que as questões alimentares podem influenciar positivamente ou negativamente na qualidade de vida dos portadores das DCNT é amplamente discutida, porém ainda não se vêem ações de valorização do profissional apto a realizar 
esse planejamento, que é o nutricionista. Sendo assim, como se apresenta neste estudo, essas ações ainda não são desenvolvidas de uma maneira global e abrangente como uma estratégia mundial ou nacional. Elas se concentram, em sua maior parte, no âmbito municipal e estadual. No caso do Brasil, talvez isso se dê pela diversidade cultural de nosso país. Portanto, o retrato epidemiológico, a necessidade de prevenção, a eficácia da Educação em saúde e para a saúde devem ser questões que impulsionem as ações dos nutricionistas mesmo frente às dificuldades em prol da melhoria da saúde e qualidade de vida da população do município.

A construção de uma cartilha, a partir da necessidade de cada município de formular ações educativas e informativas na área de nutrição, destinadas principalmente aos ACS, que atuam em cada micro área, pode otimizar as orientações prestadas no que tange a mudança no estilo de vida das populações sedentárias: hábitos alimentares que prejudicam a saúde e que, muitas vezes, se sedimentam por falta de conhecimento, instrução e por herança familiar. A cartilha poderá ser uma ferramenta de apoio para o nutricionista, no treinamento dos ACS. O objetivo deste estudo é elaborar uma cartilha de condutas nutricionais para portadores de Diabetes Mellitus e Hipertensão Arterial para usuários da ESF.

\section{REVISÃO DA LITERATURA}

\section{Diabetes e Nutrição}

São várias as razões que contribuem para muitas pessoas acreditarem que a terapia nutricional é a parte mais difícil do tratamento do Diabetes Mellitus. A começar pela mentalidade criada a partir dos mitos de que o portador de Diabetes terá uma vida repleta de restrições alimentares. Certamente, o indivíduo que recebe esse diagnóstico necessitará ajustar seu plano alimentar, que, dependendo de como se davam seus hábitos antes do diagnóstico, podem exigir ajustes grandes. Atualmente, além das pesquisas estarem avançando no campo da dietoterapia, em que o consumo de carboidratos não está entre os maiores vilões, como se achava no passado, a indústria de alimentos também cresce no que diz respeito à variedade de produtos específicos para essa população e corrobora com este pensamento Lottenberg (2008).

Entre as dificuldades citadas pelos diabéticos para seguir a terapia nutricional, pode-se citar também a não-individualização do plano alimentar, o fato de os objetivos 
do tratamento não serem realistas, porque normalmente são estabelecidos para o portador de diabetes, e não com ele, sem verificar a sua expectativa. Além disso, a falta de apoio da família e dos profissionais de saúde, bem como o despreparo técnico destes, é preponderante para o não-cumprimento do plano alimentar. A falta de motivação, a resistência às mudanças, uma vez que o paciente passou boa parte de sua vida com estilo de vida diferente e a procura de "milagres" dificultam, também, o segmento da terapia nutricional (CUPPARI, 2002). Entretanto, cada um desses pontos pode ser trabalhado para aumentar as chances de sucesso. Apesar da constatação de milagres não existentes, os profissionais de saúde devem considerar os conceitos e as crendices, os mitos e tabus e trabalhar com esses aspectos durante a orientação do plano alimentar a ser instituído. Assim, a educação para a monitoração quanto ao aspecto nutricional proposta a partir desse instrumento educacional se faz necessária para consolidar esse apoio por parte da equipe de saúde.

\section{Hipertensão e Nutrição}

Para que haja o controle da Hipertensão Arterial, sabe-se que a terapia nutricional aliada a hábitos de vida saudáveis e aos medicamentos constituem-se as medidas de maior relevância. O controle da pressão arterial, por meio de medidas dietéticas específicas, visa não apenas a redução dos níveis tensionais, mas também a incorporação de hábitos alimentares permanentes. A dietoterapia faz parte de um conjunto de medidas terapêuticas, não-farmacológicas, que têm como principal meta diminuir a morbimortalidade por meio de modificações do estilo de vida. Dessa forma, como coadjuvantes do tratamento dietético, temos outras medidas fundamentais como a redução do consumo de bebidas alcoólicas, o abandono ao tabagismo, a redução de peso corpóreo e a atividade física (CUPPARI, 2002).

Uma outra medida importante a ser adotada é a diminuição da ingestão de alimentos que contém cloreto de sódio, que há muito tempo tem sido considerado importante fator no desenvolvimento e na intensidade da hipertensão e a ingestão de sal bastante citada na literatura. As medidas terapêuticas não-farmacológicas no tratamento da hipertensão arterial fazem parte de uma mudança comportamental global. A incorporação de novos hábitos de vida garante que os benefícios alcançados sejam duradouros desde que as mudanças no estilo de vida sejam permanentes (CUPPARI, 2002). 


\section{O papel do Nutricionista na Estratégia Saúde da Família}

O trabalho do nutricionista neste campo está voltado para ações de promoção e recuperação da saúde por meio de práticas alimentares saudáveis; prevenção das carências nutricionais e das doenças crônicas não transmissíveis, tais como Diabetes, Obesidade, Neoplasias, Hipertensão Arterial e Hiperlipidemias. As principais atividades do nutricionista, que atua nesse segmento, são atendimentos ambulatoriais; visitas domiciliares; palestras educativas e reuniões voltadas para a promoção de práticas alimentares saudáveis com grupos de agentes comunitários de saúde, diabéticos e hipertensos, idosos, gestantes e mães de crianças com baixo peso.

As principais vantagens de atuar na saúde são as interações do nutricionista com outros profissionais da área, o que torna mais efetiva as ações de promoção da saúde das famílias assistidas pelas equipes da ESF, e a assistência às famílias no próprio domicilio, o que permite conhecer sua realidade, costumes, situação socioeconômica e as condições higiênico-sanitárias. Assim, o nutricionista propõe orientações nutricionais cabíveis e necessárias, adequadas às condições da família (MENON, 2008).

O CRN-4 também define que o nutricionista é o profissional habilitado para realizar ações de alimentação e nutrição voltadas para a promoção da saúde da população. Portanto, o principal desafio para essa ação consiste em atingir o maior número de famílias e sensibilizá-las para a promoção de mudanças dos hábitos alimentares frente às condições de alimentação e ao estilo de vida adotado e/ou imposto pela sociedade atual.

\section{METODOLOGIA}

Trata-se de um estudo do tipo metodológico que se refere à elaboração de instrumentos e técnicas de pesquisa (POLIT et al., 2004). Para Lo Biondo (2001), o pesquisador no desenvolvimento da pesquisa metodológica se interessa em transformar um conhecimento construído para um formato tangível. O projeto de pesquisa foi previamente aprovado pelo Comitê de Ética em Pesquisa do Centro Universitário de Volta Redonda, credenciado no Conselho Nacional de Ética em Pesquisa.

\section{A sistematização do conteúdo}


O conteúdo da cartilha foi composto tendo como base encontros com a Coordenação do Programa de Diabetes e Hipertensão Arterial da SMS de Volta Redonda. Buscou-se a aprovação do conteúdo da cartilha do ponto de vista nutricional. Houve, a partir das propostas feitas por esta coordenação, uma adequação do produto.

Na etapa seguinte, de sistematização desse conteúdo, foi realizada uma revisão de literatura para garantir a fundamentação científica, pois isto é condição essencial para preservar a segurança do leitor e definir apropriadamente os conceitos constantes em um material educativo (ECHER, 2005). Todo o processo de construção da cartilha foi permeado pela atenção voltada à adequação da linguagem. Este trabalho se caracterizou pela identificação dos termos técnicos e a transformação deles para a linguagem popular, de modo a facilitar a compreensão da cartilha por seus usuários - importante nos trabalhos relacionados à educação e promoção da saúde. Nesse sentido, devem ser preferencialmente utilizadas as palavras de uso popular, sobretudo as coloquiais (REBERTE, 2009). O emprego de termos técnicos devem se restringir ao estritamente necessário e, neste caso, os devidos esclarecimentos devem ser feitos mediante a utilização de exemplos (DOACK, apud REBERTE, 2009).

\section{A seleção das ilustrações}

Considerou-se a necessidade de incluir algumas ilustrações na cartilha, com o objetivo de tornar a leitura descontraída e de fácil compreensão (ECHER, 2005). Definiu-se que seriam incluídas ilustrações prontas, selecionadas a partir de outros materiais e que as respectivas fontes seriam citadas na cartilha. Este estudo foi realizado mediante consulta a livros-texto e imagens disponibilizadas em páginas eletrônicas.

\section{A composição da cartilha}

Para a composição da cartilha propriamente dita, foi realizado contato com um profissional de artes gráficas, que foi solicitado para realizar o trabalho de edição do material. O conteúdo a ser incluído no material educativo foi entregue a este profissional, que criou um roteiro preliminar indicativo de uma publicação. Ele também definiu as três etapas que devem ser desenvolvidas no processo de elaboração: a apresentação, as considerações sobre promoção de saúde aos portadores de Diabetes e HAS e o processo ou assunto central da publicação. 
Com base neste roteiro, foi elaborada a apresentação do material e feitas novas adequações no conteúdo da cartilha. Houve necessidade de reduzir as informações porque a inclusão de todo o conteúdo seria excessivo em se tratando de uma cartilha. Foi dada prioridade às informações relevantes, pois os textos extensos reduzem a velocidade da leitura e dificultam a compreensão das informações, por parte do leitor (DOACK, 1996, p.54 apud REBERTE, 2009). O novo conteúdo desta vez restringido foi apresentado ao profissional que fez a editoração da cartilha. Concluído este trabalho, foi feita a organização estrutural e de formato do material. A seguir, a diagramação da cartilha e sua transformação para um formato possível de impressão.

\section{RESULTADOS}

\section{Versão Final da Cartilha}

Constitui-se em um material educativo, contendo 42 páginas. Estas têm a dimensão de 210 x 297 mm, impressas nas cores: preto, azul, amarelo e vermelho escuro, sobre papel couché, presas por dois grampos. O título da cartilha é “Orientações Nutricionais para a Estratégia Saúde da Família - Diabetes e Hipertensão". O conteúdo desta cartilha contém uma apresentação inicial e, na sua sequência, os seguintes assuntos que estão apresentados na forma de tópicos: Apresentação; Entendendo o Diabetes; Principais sintomas do Diabetes; O uso de adoçantes; Entendendo a Hipertensão Arterial; Dicas para perder peso; Dicas para evitar o consumo de sal; Dicas para evitar o estresse; Os Dez passos para uma alimentação saudável; Orientações gerais; Receitas. No final da cartilha, encontra-se uma lista de referências que foram consultadas para elaborar o texto e a ilustrações.

\section{CONSIDERAÇÕES FINAIS}

O combate efetivo da HAS e do Diabetes requer estratégias de longo prazo no que diz respeito à proteção, promoção e apoio a modos de vida saudáveis, enfatizando a prática de atividade física regular e adoção de uma alimentação saudável. Como se verifica nesse estudo, a educação nutricional é um fator indispensável na prevenção e controle dessas patologias.

Entendemos que o produto Orientações Nutricionais para a Estratégia Saúde da Família - Diabetes e Hipertensão, desenvolvido neste estudo venha ajudar às equipes de saúde da Atenção Primária, principalmente o ACS, a orientar a comunidade no que diz respeito a alimentação de diabéticos e hipertensos. Trata-se de uma cartilha de 
comunicação simples e fácil acesso, que irá aperfeiçoar a educação nutricional na Atenção Básica. E tem como finalidade ratificar a necessidade da inserção do profissional nutricionista em todas as unidades da ESF, admitindo que nenhum método possua a chave do sucesso perfeito. Trata-se de uma ferramenta pedagógica de orientação que busca agregar conhecimento aos agentes para aproximá-los dos usuários de seu território através de informações fundamentais para o controle das DCNT, desmistificando conceitos equivocados acerca da dieta, estimulando as motivações do ACS para compartilhar projetos e soluções para a comunidade de maneira contínua, gerando assistência na vida comunitária e tendo como princípio a vigilância e promoção à saúde, por meio de sua forte identificação com a comunidade, o que o constitui um trabalhador singular em saúde.

\section{REFERÊNCIAS}

BATISTA FILHO et al. Transição nutricional: conceito e características. In: Epidemiologia Nutricional. orgs. Kac G, Sichieri R e Gigante DP. Rio de Janeiro: Ed. Fiocruz/ Ateneu, 2007.p.445-446.

BRASIL. Departamento de Atenção Básica (DAB). Saúde da Família. Disponível em: http://dtr2004.saude.gov.br/dab/atencaobasica.php. Acesso em: setembro de 2008.

Conselho Federal de Nutricionistas (CFN), disponível em:

<http://www.cfn.org.br/novosite/arquivos/O_Papel_do_Nutricionista_na_Atencao_Prim aria_a_Saude.pdf> ; acesso em 26 de fevereiro de 2010.

CUPPARI, Lilian. Guias de medicina ambulatorial e hospitalar. UNIFESP/ Escola Paulista de Medicina: nutrição. São Paulo: Manole, 2002. p. 299-305.

ECHER,Isabel Cristina. Elaboração de Manuais de Orientação para o Cuidado em Saúde. Rev Latino-am Enfermagem: relato de caso 13(5). [online] set/out. 2005. Disponível em: http:// www.eerp.usp.br/rlae> acesso em 21 mar. 2010.

IBGE E MINISTÉRIO DA SAÚDE. Pesquisa de orçamento das famílias: 2002-2003: análise da disponibilidade domiciliar de alimentos e do estado nutricional no Brasil (POF). Rio de Janeiro, 2006.

Lo BIONDO, WG; HABER, J. Pesquisa em Enfemagem: métodos, avaliação crítica e utilização. $4^{\text {a }}$ ed. Rio de Janeiro. Guanabara Koogan; 2001.p.117.

LONGO, Giana Zarbato et al . Prevalência de níveis pressóricos elevados e fatores associados em adultos de Lages/SC. Arq. Bras. Cardiol., São Paulo, v. 93, n. 4, oct. 2009 . Disponible en <http://www.scielo.br/scielo. >. acesso em 26 feb. 2010. 
LOTTENBERG, Ana Maria Pita. Características da dieta nas diferentes fases da evolução do diabetes melito tipo 1. Arq Bras Endocrinol Metab, São Paulo, v. 52, n. 2, Mar. 2008 . Available from <http://www.scielo.br/scielo. access on 19 Mar. 2010.

MENON, P.G.V. Atuação do Nutricionista na Estratégia Saúde da Família. Revista do Conselho Regional de Nutrição-CRN 4. Rio de Janeiro e Espírito Santo. n.7,3-15, Dezembro, 2008.

PASSOS, VM, ASSIS TD, BARRETO SM. Hipertensão arterial no Brasil: estimativa de prevalência a partir de estudos de base populacional. Epidemiol Serv Saude. 2006;15(1):35-45.

POLIT, Denise F.; BECK, Cheryl Tatano; HUNGLER, Bernadette P. Fundamentos de

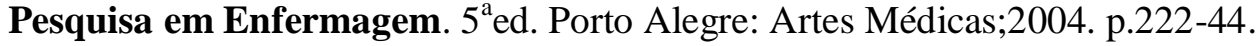

REBERTE, Luciana Magnoni. Celebrando a vida: construção de uma cartilha para promoção da saúde da gestante. 2008. 130f. Dissertação (Mestrado em Enfermagem) - Escola de Enfermagem da Universidade de São Paulo, São Paulo, 2009.

ROSA RS, Schmidt MI. Diabetes Mellitus: magnitude das hospitalizações na rede pública do Brasil, 1999-2001. Epidemiol Serv Saude. 2008;17(2):131-4.

WILD S, ROGLIC G, GREEN A, SICREE R, King H. Global prevalence of diabetes: estimates for the year 2000 and projections for 2030. Diabetes Care. 2004;27(5):104753. 